\title{
Modelisation Hydraulique Mono Dimensionnel Par HEC RAS, Application Sur L'oued Aggay (Ville De Sefrou)
}

\author{
Meriam Lahsaini, \\ Hassan Tabyaoui, \\ Laboratoire Ressources Naturelles et Environnement, Faculté \\ Polydisciplinaire de Taza, Université Sidi Mohamed Ben Abdellah, \\ Fès, Morocco
}

Doi: 10.19044/esj.2018.v14n18p110 URL:http://dx.doi.org/10.19044/esj.2018.v14n18p110

\begin{abstract}
The city of Sefrou, because of its geographical position, its cultural heritage and urban planning, than economically, is classified as one of the sites with a vulnerability particular to floods. Oued Aggay, the subject of this study, constitutes a danger potential because of the violence of its floods. In this perspective that comes this study that part of the creation and management of a spatial database on flood risk in the Sebou basin. It aims to spatialize the extent of the floods of Oued Aggay and propose solutions to protection the city of Sefrou against floods. The chosen approach goes through a hydrological study, the choice of profiles and the construction of onedimensional model from HEC RAS hydrology software. This study allowed us to simulate floods by statistical methods, identify flood zones and determine the different water levels in the flooded area for the Oued Aggay watershed.
\end{abstract}

Keywords: Hydraulic Modeling, HEC-RAS, Flood, Oued Aggay, Morocco

\section{Résumé}

La ville de Sefrou, de part sa position géographique, son patrimoine culturel et urbanistique, que sur le plan économique, est classée parmi les sites ayant une vulnérabilité particulière aux inondations. L'Oued Aggay, sujet de la présente étude, constitue un danger potentiel à cause de la violence de ses crues. Dans cette optique que vient cette étude qui s'inscrit dans le cadre de la création et la gestion d'une base de données spatiale sur les risques d'inondation dans le bassin de Sebou. Elle a pour but de spatialiser l'étendue des crues de l'Oued Aggay et proposer des solutions pour la protection de la ville de Sefrou contre les inondations. L'approche choisit passe par une étude hydrologique, le choix des profils et la construction de modèle mono- 
dimensionnel à partir de logiciel d'hydrologie HEC RAS. Cette étude nous a permis de simuler les crues par des méthodes statistiques, d'identifier les zones inondables et de déterminer les différentes hauteurs d'eau dans la zone inondée pour le bassin versant d'Oued Aggay.

Mots-clés: Modélisation hydraulique, HEC-RAS, Crue, Oued Aggay, Maroc

\section{Introduction}

$\mathrm{Au}$ cours des dernières années, de graves inondations ont eu lieu au Maroc causant des pertes économiques et de décès. La ville de Sefrou n'échappe pas à cette règle puisqu'elle est exposée au risque des quatre crues majeures de l'Oued Aggay, enregistrées en 1953, 1976, 1994, 2010 (DRPE, 2011).

Les inondations dans le Bassin versant d'Aggay prennent un caractère beaucoup plus violent et localisé, par rapport aux grandes crues des plaines. Il est certain que la progression de l'habitat construit dans les zones inondables contribue beaucoup à l'aggravation des risques et des dommages constatés lorsque survient une crue. Cependant, malgré les drames vécus, les inondations sont rapidement oubliées par les riverains eux-mêmes que par les aménageurs au profit de la sécheresse qui frappe le pays avec des récurrences beaucoup plus rapprochées et des conséquences économiques et sociales beaucoup plus fortes. Le diagnostic de la situation existante a permis de comprendre la problématique posée par les crues de l'oued Aggay.

C'est dans cette optique que vient cette étude qui a pour objectif de modéliser et spatialiser ces crues, qui touchent la ville de Sefrou, une étude hydrologique suivit par une Modélisation hydraulique par le logiciel HECRAS (USAC, 1997) est entretenu dans le bassin versant de l'Oued Aggay. Ce logiciel s'est déjà montré très performant pour ce type d'étude, et un grand nombre d'entreprises et de laboratoire de recherche le considèrent comme un outil de première approche. Tout ceci s'inscrit sous un objectif final permettant, une protection contre les inondations des zones de débordement. 
Figure 1 : Situation du Bassin versant d'Oued aggay

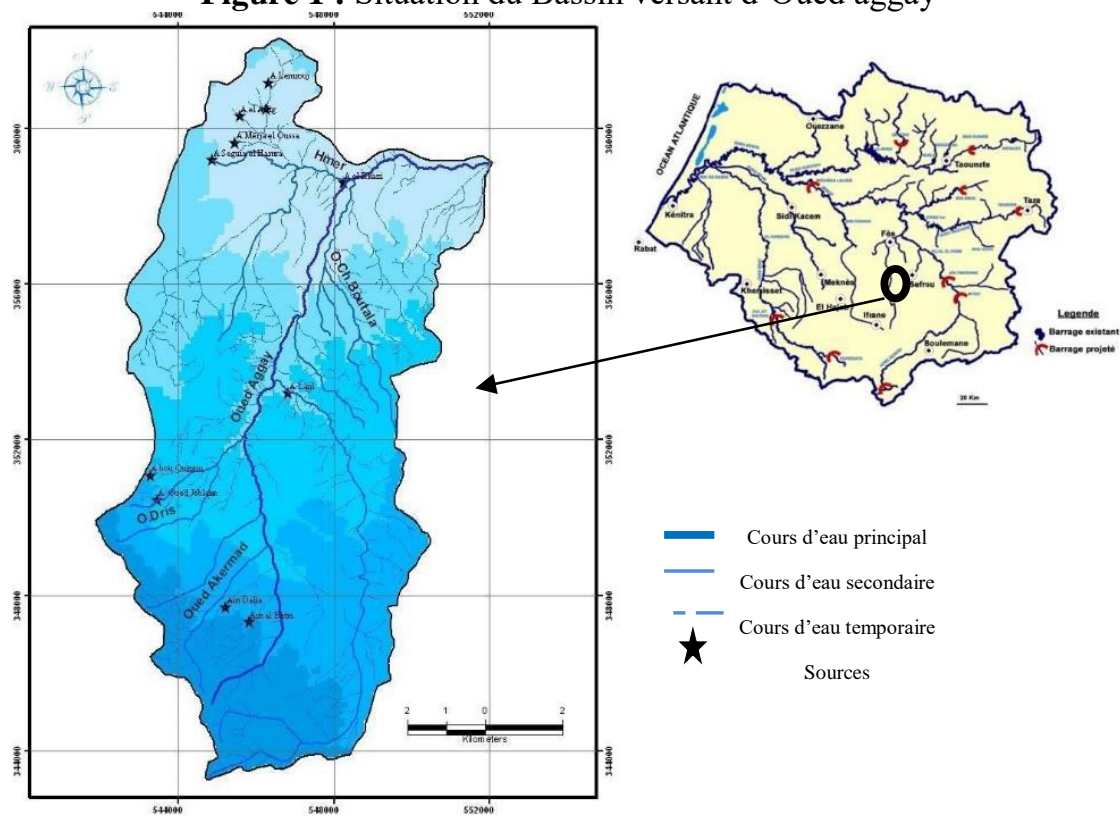

\section{Zone d'étude}

Le bassin de l'Oued Aggay est situé au SE du basin hydrologique de Sebou (fig. 1) sur le piémont du Causse moyen-atlasique. Il couvre une superficie de $108 \mathrm{~km}^{2}$ (tab.1). L'oued Aggay qui constitue le drain principal du bassin à une longueur de $26.11 \mathrm{~km}$ est progressivement alimenté tout au long de son cours par de petites sources (fig. 3). Son débit à l'étiage est faible, malgré la superficie appréciable de son bassin versant. Le climat est de type continental (hiver pluvieux et été chaud et sec). La pluviométrie est très irrégulière d'une année sur l'autre. La moyenne des précipitations est $553 \mathrm{~mm}$. Elle varie de $450 \mathrm{~mm}$ à $600 \mathrm{~mm}$. La neige apparait sur les reliefs élevés du Sud ouest à l'est du bassin. Le bassin versant a un drainage de faible densité. La valeur calculée selon la loi d'Horton (1945) est de 0.16, La pente moyenne du bassin versant est de 3.3\% et évoque un rugissement très élevé (fig. 2). L'aval du bassin versant d'Oued Aggay est connue par des roches karstfiables au piémont du Moyen-Atlas (fig. 4) et par des vallées sèches réceptacles d'averses qui constituent des zones d'empreints riches en matériaux de crues (AMRAOUI F., 2005).

La couverture végétale potentielle est généralement sylvatique (fig. 5). La biomasse forestière protectrice est parfois très réduite. Le ruissellement superficiel favorise le décapage du sol et le démantèlement des terrasses au fond des vallées. La très forte densité d'urbanisation au centre de la ville de Sefrou, un habitat diffusé au Nord et au Sud du BV, se faisant souvent en lit majeur des criques et dans les zones altimétriquement basses. 
L'action combinée de l'anthropisme et des facteurs naturels a intensément fragilisé le bassin versant d'Oued Aggay. Cette double fragilisation à l'aval du BV et l'expansion urbaine exposée au risque d'inondation ont fait de la ville de Sefrou un site dont les problèmes d'aménagement sont épineux, nécessitant des outils de planification adéquats et des interventions coordonnées et efficaces en matière d'aménagement.

Tableau 1 : Paramètres physiographique du bassin versant d'Oued Aggay

\begin{tabular}{|c|c|c|c|c|c|c|c|c|c|}
\hline \multirow{2}{*}{$\begin{array}{l}\text { Bassin } \\
\text { versant }\end{array}$} & \multirow{2}{*}{$\begin{array}{l}\text { Surface } \\
(\mathrm{km} 2)\end{array}$} & \multirow{2}{*}{$\begin{array}{l}\text { Périmètre } \\
(\mathrm{Km})\end{array}$} & \multicolumn{2}{|c|}{$\begin{array}{c}\text { Indice de Gravilius } \\
\text { Kc }\end{array}$} & \multirow{2}{*}{$\begin{array}{l}\text { Altitude } \\
\max (\mathrm{m})\end{array}$} & \multirow{2}{*}{$\begin{array}{l}\text { Altitude } \\
\text { min }(m)\end{array}$} & \multirow{2}{*}{$\begin{array}{l}\text { Pente } \\
\text { moy } \\
(\%)\end{array}$} & \multicolumn{2}{|c|}{$\begin{array}{l}\text { Cours d'eau } \\
\text { principale }\end{array}$} \\
\hline & & & Valeur & Forme & & & & Nom & $\begin{array}{c}\text { Longueur } \\
(\mathrm{Km})\end{array}$ \\
\hline $\begin{array}{l}\text { Oued } \\
\text { Aggay }\end{array}$ & 108 & 56 & 1.49 & Allongée & 1735 & 880 & 3.29 & $\begin{array}{l}\text { Oued } \\
\text { Aggay }\end{array}$ & 26.11 \\
\hline
\end{tabular}

Figure 2: carte de pentes

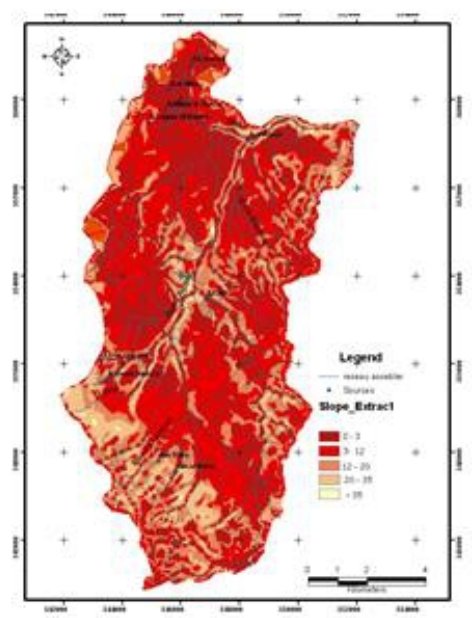

Figure 4: carte géologique

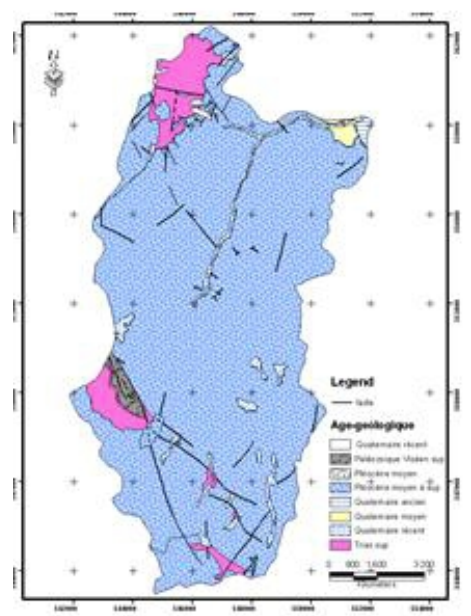

Figure 3: carte de réseau hydrographique

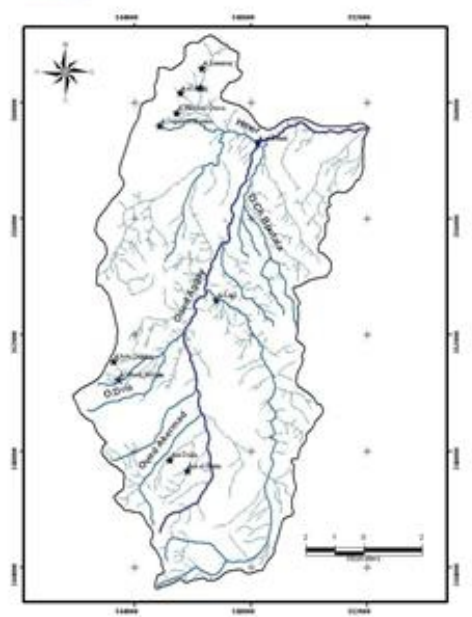

Figure 5: carte d'occupation des sols

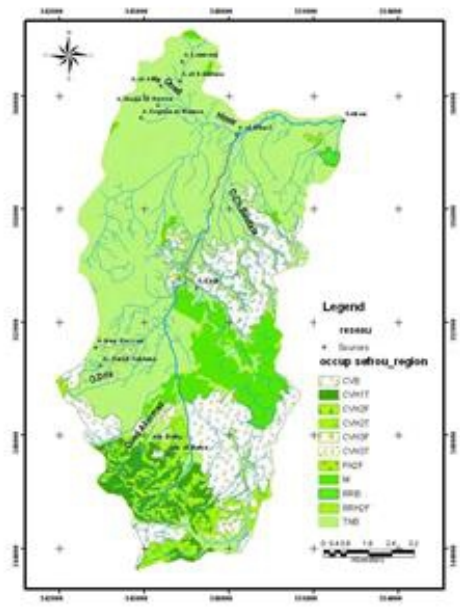




\section{Méthodologie}

La modélisation s'avère un outil fondamental pour connaitre la répartition et l'ampleur géographique du phénomène. Notre schéma de modélisation et de mesure de l'ampleur d'inondation est fondé sur la base de deux études, la première est hydrologique et a pour objectif l'estimation des débits de pointes correspondant aux différentes périodes de retour au niveau de la zone du projet. Les débits adoptés seront d'une importance capitale pour le dimensionnement des ouvrages projetés sur 1'oued Aggay. Elle comprend plusieurs étapes :

- Trouver des laisses de crues passés et recueillir les témoignages des habitations aux endroits les plus pertinents. De ce fait la ville de Sefrou est exposée aux inondations dans les plus importantes sont cette de 1950-19921994.

- $\quad$ Ajustement par loi de Gumbel des pluies maximales journalières et détermination des $\mathrm{Pj} \max (\mathrm{T})$ pour une période $\mathrm{T}$ allant de 10 à 100. Cette méthode s'applique à des bassins de quelques dizaines à plusieurs milliers de kilomètres carrés, qui sont homogènes pluviomètriquement.

- $\quad$ Estimation des débits de crue par la méthode de Gradex modifiée cette méthode a été élaborée dans le cadre de l'étude du Plan Directeur intégré d'aménagement des eaux des bassins Sebou.

- $\quad$ Estimation du débit de pointe par les différentes formules empiriques (Mallet Gauthier, Fuller II, Fuller I, HazenLazarevich) qui consiste à appliquer les formules régionales adaptées à la nature du bassin versant. Elles tiennent compte la taille et le gradient topographique du bassin versant.

- $\quad$ Estimation des temps de concentration en appliquant les formules empiriques.

- $\quad$ Estimation de l'hydrogramme de crues.

La seconde phase est la phase de la simulation d'un modèle d'oued Aggay. Elle commence par apporter les éléments de bases nécessaires au fonctionnement du modèle hydraulique, et identifier des zones à risques d'inondations dans le bassin. L'identification des processus est faite en quatre étapes:

- Identification de la géométrie d'un cours d'eau (création de modèle numérique de terrain d'une grande précision altimétrique à partir de fonds topographiques. Ce modèle est généré à partir de courbes de la carte topographique de Sefrou au 1/50000); et les données relatives à chaque session (détermination du coefficient de Manning selon la nature du type du sol à partir des observations sur le terrain).

- Identification des débits et les conditions aux limites.

- Identification des conditions du calcul (hauteur d'eau, profondeur critique... ou hydrogramme, limnigramme...selon le régime d'écoulement). 
- Visualisation des résultats sous forme graphique ainsi que sous forme des tableaux.

Pendant cette étude, nous avons utilisé deux logiciels : un logiciel de Système d'Information géographique (Arc-Gis) et un logiciel de modélisation de rivières (HEC-RAS) qui est développé au Hydrologic Engineering Center de l'US Army Corps of Engineers (USAC, 1997). Les calculs de lignes d'eau d'écoulements graduellement variés sont basés sur l'équation de Bernoulli. Les pertes de charge sont évaluées par l'équation de Manning-Strickler pour le terme de frottement et par des coefficients de contraction-expansion. En écoulement brusquement varié, HEC-RAS utilise l'équation de quantité de mouvement.

Figure 6: Schéma méthodologique

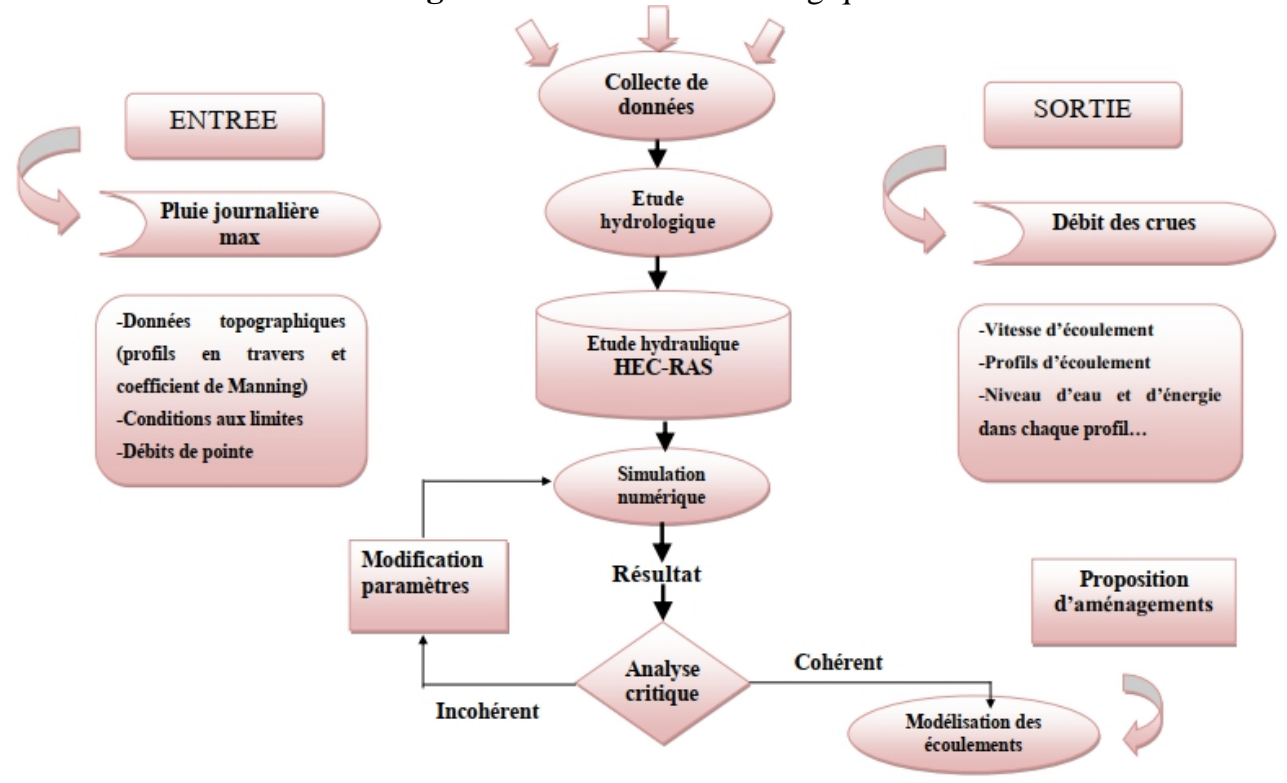

\section{Résultats et discussions}

\section{$>\quad$ Etude hydrologique}

Ajustement des pluies journalières par loi de Gumbel : L'application de la méthode Gradex a permis d'ajuster les séries de pluies maximales et les débits correspondants. Les valeurs représentées dans le tableau correspondent à des valeurs journalières. Le passage aux pluies en 24 $\mathrm{h}$, se fera en multipliant les valeurs par un coefficient égal à 1.15 et ceci pour tenir compte de la coulissante de la pluie (tabl. 2). La valeur de Gradex doit aussi être changée avec la multiplication de Gradex du pluies journalières par la même coefficient de 1.15 . 
Tableau 2 : P24 de chaque période de retour avec le Gradex

\begin{tabular}{|c|c|c|c|c|c|}
\hline Station & Gradex 24 h & $\begin{array}{c}\text { Pjmax } \\
10 \text { ans }\end{array}$ & $\begin{array}{c}\text { Pjmax } \\
20 \text { ans }\end{array}$ & $\begin{array}{c}\text { Pjmax } \\
50 \text { ans }\end{array}$ & $\begin{array}{c}\text { Pjmax } \\
100 \text { ans }\end{array}$ \\
\hline Sefrou & 10.7 & 71.98 & 80 & 89.64 & 97.10 \\
\hline
\end{tabular}

Méthode de Gradex modifié: elle a été élaborée dans le cadre de l'étude du Plan Directeur intégré d'aménagement des eaux des bassins Sebou, l'application de cette méthode a permis de'estimer les débits de crue. Le débit de pointe Qp est donné par la relation suivante : $\mathrm{Qp}(\mathrm{T})=\mathrm{Cp} * \mathrm{Qj}(\mathrm{T})$

Avec: Qp :débit de pointe (m3/s) ;

Qj :débitmoyen journaliér (m3/s) ;

$\mathrm{Cp}$ :coefficient de pointe. $\mathrm{Cp}=3.08$

Tableau 3: débit estimés par la methode de Gradex

\begin{tabular}{|c|c|c|c|c|}
\hline & T10 & T20 & T50 & T100 \\
\hline Qp (m3/s) & 100 & 120 & 170 & 210 \\
\hline
\end{tabular}

Estimation du débit de pointe par des formules régionales: Les débits retenus à partir de l'analyse et la comparaison des débits calculés par les différentes méthodes (Mallet Gauthier, Fuller II, Fuller I, Hazen Lazarevich) sont synthétisés dans le tableau 4 :

Tableau 4: débit estimés par différentes méthodes régionaux

\begin{tabular}{|c|c|c|c|c|}
\hline & \multicolumn{4}{|c|}{ Débit (m3/s) } \\
\hline & $\begin{array}{c}\text { Mac } \\
\text { Math }\end{array}$ & $\begin{array}{c}\text { Hazen } \\
\text { Lazarevich }\end{array}$ & $\begin{array}{c}\text { Fuller } \\
\text { II }\end{array}$ & $\begin{array}{c}\text { Mallet } \\
\text { Gauthier }\end{array}$ \\
\hline T10 & 233.9 & 126.16 & 142.90 & 173.24 \\
\hline T20 & 259 & 143.04 & 162.02 & 205.51 \\
\hline T50 & 291 & 165.36 & 187.3 & 241.57 \\
\hline T100 & 316 & 182.23 & 207.19 & 265.62 \\
\hline
\end{tabular}

Calcul du temps de concentration: Le temps de concentrationtc des eaux sur un bassin versant se définit comme le maximum de durée nécessaire à une goutte d'eau pour parcourir le chemin hydrologique entre un point du bassin et l'exutoire de ce dernier. Le temps de concentration s'estime à l'aide des formules les plus répandues au Maroc (Formules de Turazza, Ventura, Kripich, D. Californienne, H. Giandotti, Passini), le temps de concentration du bassin versant d'oued Aggay est proche de 3h (tabl. 5). Nous notons que ce temps de concentration est relativement court ce qui peut être expliqué par la forme allongée du bassin et les pentes importantes qui y règnent. 
Tableau 5 : Tc par différents méthodes appliquées au bassin de l'oued Aggay

\begin{tabular}{|c|c|c|c|c|c|}
\hline 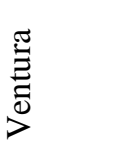 & : & 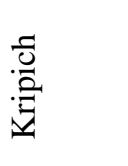 & 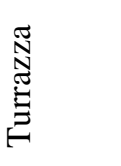 & 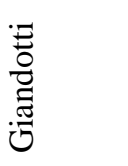 & Valeur retenue $(\mathrm{h})$ \\
\hline $7.27 \mathrm{~h}$ & $3 \mathrm{~h}$ & $3.04 \mathrm{~h}$ & $8.39 \mathrm{~h}$ & $4.88 \mathrm{~h}$ & $3 \mathrm{~h}$ \\
\hline
\end{tabular}

l'hydrogramme de crue: pour définir un hydrogramme type des crues d'un oued, on «adimensionalise» les crues les plus représentatives inventoriées par leurs temps de pointe et débits de pointe et on les superpose aux hydrogrammes type. On adopte L'hydrogramme de l'USSCS, il s'agit d'un hydrogramme type adimensionnel (fig. 7) avec comme données en entrée le temps de base et le débit de pointe. Le temps de monté égale à $3 \mathrm{~h}$ et le temps de base égale à 3 fois le temps de monté.

Figure 7 : Hydrogramme des crues du bassin versant de l'oued Aggay

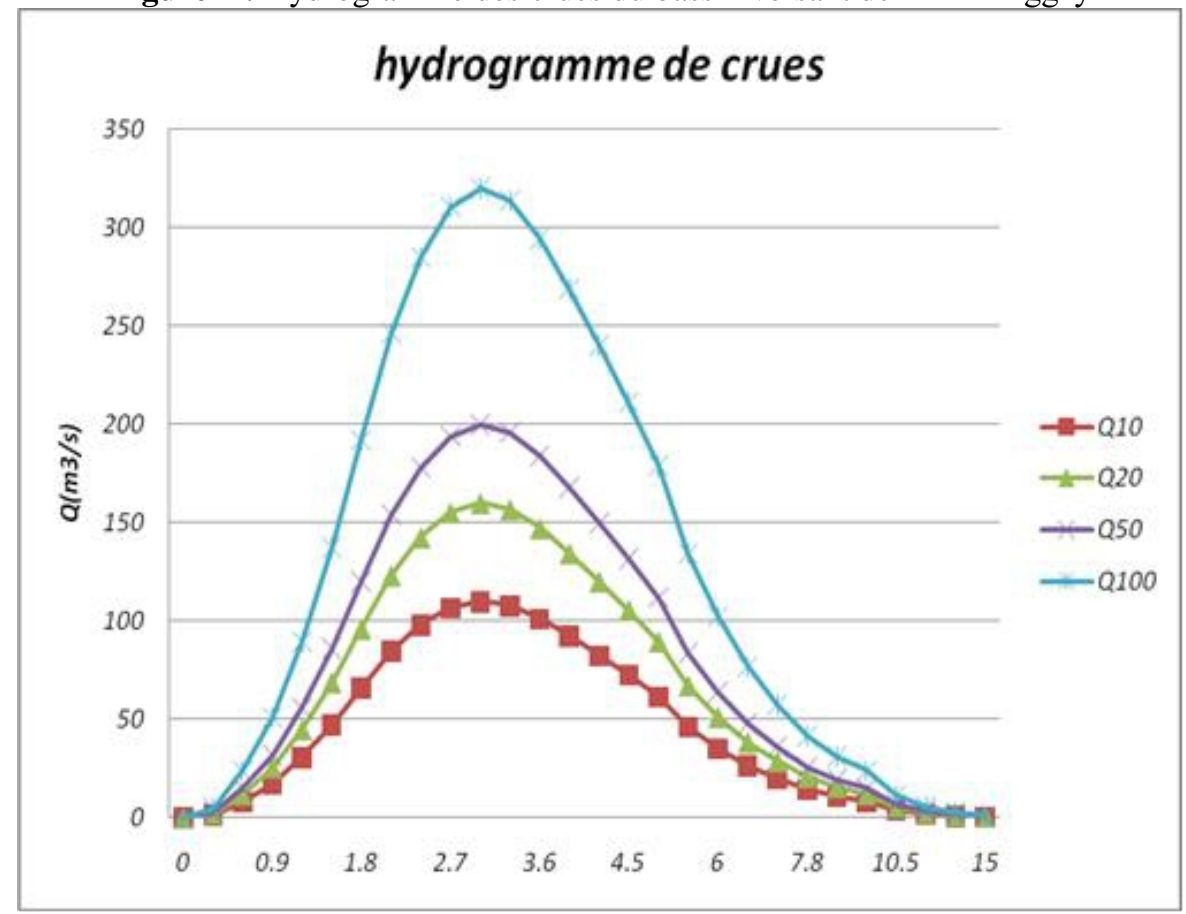

\section{Etude hydraulique}

le régime permanent reste le plus simple et le plus satisfaisant pour la réalisation du modèle hydraulique d'oued Aggay et pour savoir des résultats exploitables dans l'étude du bassin versant. On a construit un modèle hydraulique de l'oued Aggay au niveau de l'aval du barrage Aggay en régime permanent. 
Profils en travers : Les données topographiques constituent le socle sur lequel se construisent toutes les modélisations hydrauliques de rivières. Environ 100 profils topographiques répartis sur 5 kilomètres de cours d'eau ont été levées. Les profils choisis sont perpendiculaires à la direction d'écoulement des eaux, ne se croisent pas entre eux, recoupent toute la plaine alluviale et tiennent compte des modifications géomorphologiques du lit majeur. Ils sont situés à l'aval du barrage Aggay en régime permanent.

Coefficient de Manning : Le coefficient de rugosité du lit permet d'avoir une modélisation la plus proche de la réalité en prenant en compte la vitesse de l'eau sur le fond de l'Oued et sur les rives. Sur chaque profil en travers le coefficient de Manning sera fixé à 0,033 pour les rives de l'Oued Aggay. Ce qui correspond à la valeur normale pour une rivière de largeur moyenne de 10 mètres, composée d'une granulométrie grossière (galets) avec présence d'une végétation rivulaire. Les autres valeurs indicatives du coefficient de Manning, pour les autres portions de l'Oued Aggay sont présentées sur le tableau 6.

Tableau 6 : les valeurs indicatives du coefficient de rugosité

\begin{tabular}{|c|c|}
\hline Les rives & 0.033 \\
\hline Lits bétonnés & 0.015 \\
\hline Lits naturels avec vegetation & 0.05 à 0.1 \\
\hline
\end{tabular}

Débits retenus : Les débits retenus Qp à partir de l'étude hydrologique, sont les résultats d'une analyse et une comparaison des débits calculés pour différentes périodes de retour.

Tableau 7 : Débits des périodes de retour nécessaires à la modélisation

\begin{tabular}{|c|c|c|c|c|}
\hline & T10 & T20 & T50 & T100 \\
\hline $\mathrm{Qp}(\mathrm{m} 3 / \mathrm{s})$ & 112.24 & 165.91 & 211.04 & \\
\hline
\end{tabular}

Conditions aux limites : les données hydrologiques sont les conditions aux limites, pour la simulation en régime permanent, le débit est constant, la simulation HEC-RAS ne considère qu'un quel débit dans la rivière. La condition avale imposée au modèle est une cote connue du niveau d'eau égale à $2 \mathrm{~m}$.

Calage et validation : Le modèle a été calé à partir de la crue du 1994, dernière crue significative d'oued Aggay. Le calage d'un modèle se fait après plusieurs simulations en faisant varier le coefficient du Manning Strickler.

Les résultats de cette simulation sont affichés sous forme de graphique (fig. 8) Cette vue en trois dimensions montre que dans la partie amant située au sud du bassin, il n'y a pas un fort débordement de crue. A cause de la construction du barrage Aggay, la concentration des eaux s'est effectuée au 
centre de la vallée. Dans la partie avale, s'observe un énorme débordement et surtout dans la partie gauche. Ce débordement touche l'amont de la ville de sefrou.

Figure 8 : vue du tronçon modélisé en 3D

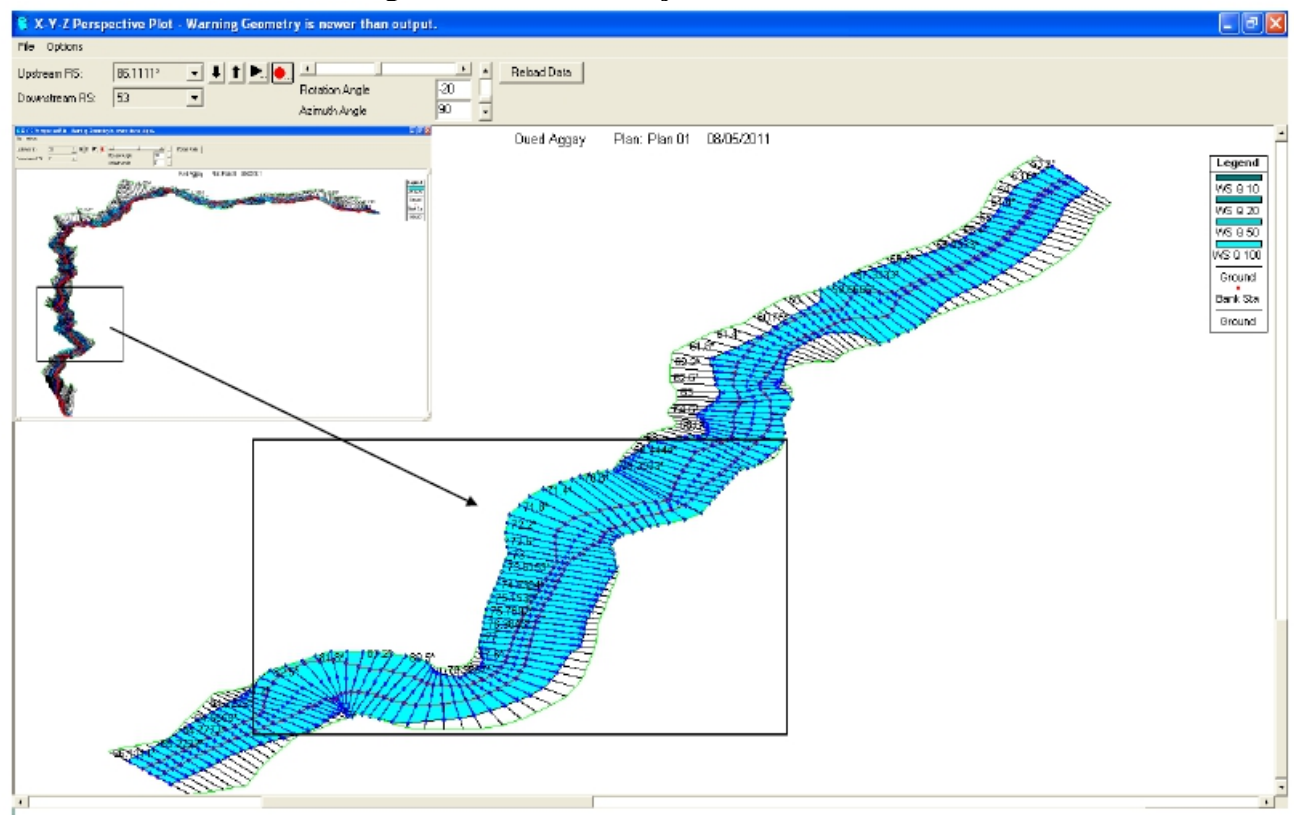

La figure 9 représente la variation de vitesse d'écoulement des eaux pour chaque période de retour. On observe presque la même allure pour tous les niveaux d'eau. La vitesse augmente avec le niveau d'eau. La variation de vitesse est fonction de la topographie de terrain : en aval (à $5240 \mathrm{~m}$ à partir de l'exutoire), La vitesse moyennement variable et de faible valeur, devient importante $(6.61 \mathrm{~m} / \mathrm{s})$ surtout le long des tronçons situées à des distances entre $4800 \mathrm{~m}$, et $5200 \mathrm{~m}$ de l'exutoire. Ces endroits sont caractérisés par une pente assez importante. Finalement, on distingue presque une même vitesse à gauche et à droite de la rivière et qui est plus faible que la vitesse au centre. La vitesse d'écoulement influence directement sur l'importance de l'érosion et la quantité de volume transporté par les eaux de crue ainsi que la force de pénétration des eaux dans les domaines urbains et leur destruction. 
Figure 9: Variation de la vitesse d'écoulement en fonction de la distance à partir de l'exutoire

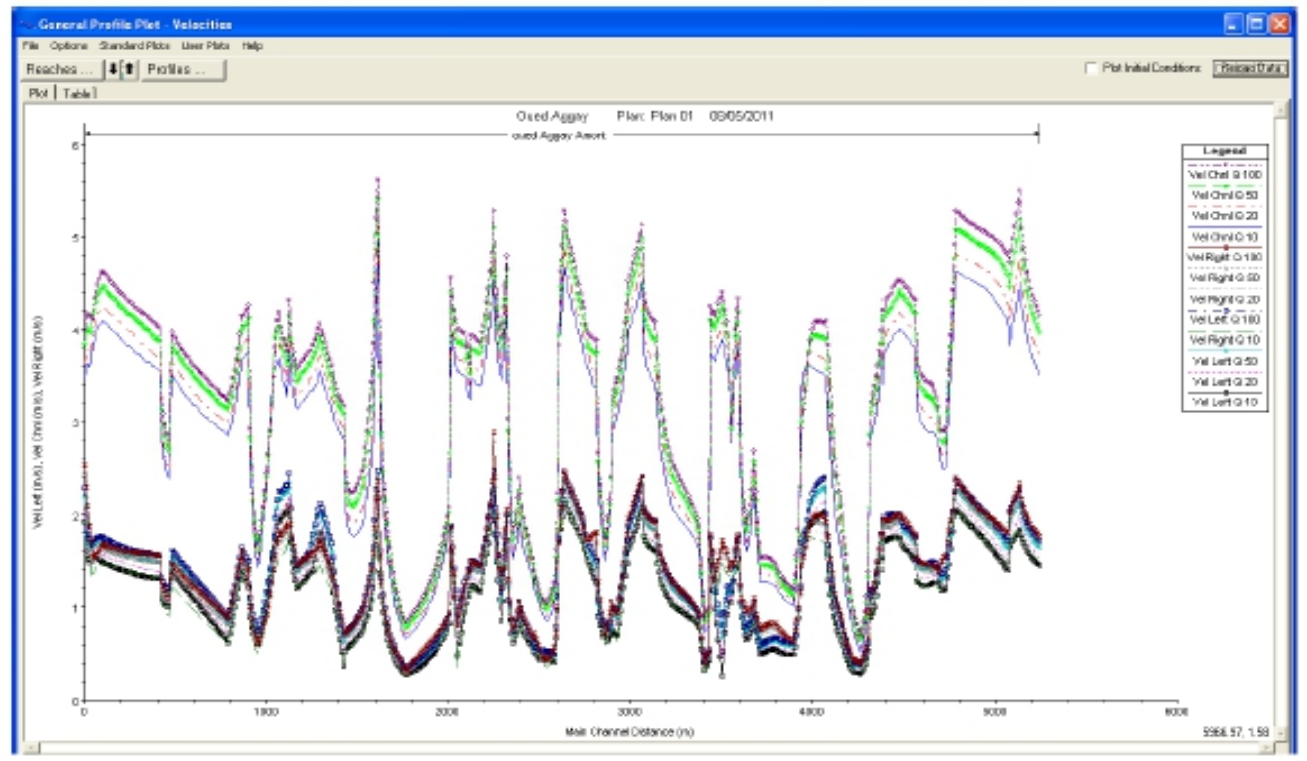

\section{Conclusion}

Cette étude permet de déterminer les zones de débordement dans le bassin versant d'Oued Aggay. La modélisation hydraulique à une dimension, grâce au logiciel HEC-RAS, permet d'une part la simulation de crue à différentes périodes de retour, et d'autre part de voir l'évolution hydraulique de l'oued Aggay en utilisant les hauteurs, les énergies et les vitesses de l'écoulement.

Les trois principales crues qu'a connues la ville de Séfrou (1950-19761994) reflètent le danger potentiel que constitue Oued Aggay. Ceci incite à la prudence en ce qui concerne l'aménagement dudit oued. Il y a lieu de signaler que la protection contre les inondations ne passera pas que par les travaux d'aménagement, mais aussi par des mesures de prévision et de prévention, souvent moins coûteuses. Ces dernières concernent la cartographie des zones à risques d'inondations, la réglementation de l'occupation des sols, l'entretien des cours d'eau et surtout l'aménagement des bassins versants.

\section{References:}

1. Amraoui F., 2005. Contribution à la connaissance des aquifères karstiques : cas su Lias de la plaine du Sais et du Causse Moyen Atlasique tabulaire (Maroc), Thèse de Doctorat d'État. Université Hassan II-Ain Chock. 237 p 
2. DRPE, 2011. Etude pour la réalisation d'une cartographie et d'un Système d'Information Géographique sur les risques majeurs au Maroc, Mission 1 Identification des risques.

3. Horton, R. E., 1945. Erosional development of streams and their drainage basins (hydrophysical approach to quantitative morphology): Geological Society of America Bulletin, v. 56. P.275-390.

4. USACE, 1997. HEC-RAS River Analysis System, Hydraulic Reference Manual, US Army Corps of Engineers.

5. USER'S Manuel HEC, (2002): guide de HEC RAS (detailed description of each of major modeling compents, and how to view graphical and tabular output.

6. Us Army Corps of Engineers, Hydrologic Engineering Center HEC GeoRAS, GIS Tools for support of HEC RAS using Arc Gis. User's manual. [PDF téléchargeable]. USACE, Version 4, Septembre 2005.

7. Us Army Corps of Engineers, Hydrologic Engineering Center. HECHMS Hydrologic modeling system. Useras manuel. [PDF téléchargeable]. USACE, Version 3.0.0, Décembre 2005.http://www.hec.usace.army.mil/software/hechms/documentatio n/CPD-74A_2005Dec.pdf. 